\title{
Experimental Issues in Testing a Semiactive Technique to Control Earthquake Induced Vibration
}

\author{
Nicola Caterino, ${ }^{1}$ Mariacristina Spizzuoco, ${ }^{2}$ Julian M. Londoño, ${ }^{2}$ and Antonio Occhiuzzi ${ }^{1}$ \\ ${ }^{1}$ Department of Engineering, University of Naples "Parthenope", Centro Direzionale di Napoli, Isola C4, 80143 Naples, Italy \\ ${ }^{2}$ Department of Structures for Engineering and Architecture, University of Naples "Federico II", Naples, Italy \\ Correspondence should be addressed to Nicola Caterino; nicola.caterino@uniparthenope.it
}

Received 31 May 2013; Revised 14 April 2014; Accepted 17 April 2014; Published 14 May 2014

Academic Editor: Nikos D. Lagaros

Copyright (c) 2014 Nicola Caterino et al. This is an open access article distributed under the Creative Commons Attribution License, which permits unrestricted use, distribution, and reproduction in any medium, provided the original work is properly cited.

\begin{abstract}
This work focuses on the issues to deal with when approaching experimental testing of structures equipped with semiactive control (SA) systems. It starts from practical experience authors gained in a recent wide campaign on a large scale steel frame structure provided with a control system based on magnetorheological dampers. The latter are special devices able to achieve a wide range of physical behaviours using low-power electrical currents. Experimental activities involving the use of controllable devices require special attention in solving specific aspects that characterize each of the three phases of the SA control loop: acquisition, processing, and command. Most of them are uncommon to any other type of structural testing. This paper emphasizes the importance of the experimental assessment of SA systems and shows how many problematic issues likely to happen in real applications are also present when testing these systems experimentally. This paper highlights several problematic aspects and illustrates how they can be addressed in order to achieve a more realistic evaluation of the effectiveness of SA control solutions. Undesired and unavoidable effects like delays and control malfunction are also remarked. A discussion on the way to reduce their incidence is also offered.
\end{abstract}

\section{Introduction}

Semiactive (SA) structural control is widely considered the prominent emerging technology for the seismic protection of civil structures. It exploits special devices, in many cases dampers, whose dynamic properties can be adjusted in a small amount of time. This is done in accordance with either the characteristics of the seismic input or the instantaneous behaviour of the structure hosting the "smart" devices. Many authors agree that SA structural control performs close to those of fully active control systems. These, however, need control power on the threshold or beyond the currently available technology and, due to the mass involved in civil structures, rely on huge electric powers in the moment when power outages are most likely to happen (e.g., during earthquakes, severe storms, etc.). Conversely, SA dampers can be fed by low power electric current and, generally speaking, can be designed as fail-safe devices.

Dampers based on magnetorheological (MR) fluids represent the most promising SA devices, because the microsized particles of magnetic material dispersed in the viscous fluid inside are able to create in few milliseconds columnar structures that generate orders-of-magnitude viscosity changes and require a minimum shear stress to initiate the flow. This shear force depends on the intensity of the magnetic field where the fluid is immersed in.

In recent years, big efforts have been dedicated to the modelling of MR dampers: important results have been obtained by Guo et al. [1], Caterino et al. [2], and LozoyaSantos et al. [3]. Nevertheless this paper aims at emphasizing the importance of the experimental assessment of SA control systems based on MR dampers. Rather than an evaluation based only on numerical simulations, our interest is to show how many problematic issues likely to happen in real applications are present when experimentally testing SA systems. This paper highlights these aspects and illustrates how they can be addressed in order to achieve a more realistic evaluation of the effectiveness of SA control systems.

The loop in control applications that use SA devices consists of three basic parts: (1) an acquisition component 
including sensors and conditioners; (2) a processing and control logic component represented by the digital controller along with the dedicated software and control algorithms; (3) a command's delivering component where the real time calibration of SA devices is commanded.

Preumont [4] focuses the attention on problems related to the digital implementation of active control tests. This work points out a number of fundamental issues that have to be considered with care when processing digital signals. One of them is the computational delay as an effect of sampling, always present between the access to the computer through the analog-to-digital converter and the output of the control law. This delay depends on the way the control algorithm is implemented and on the length of the computations within the sampling period. Another issue discussed is the use of digital filters, to be carefully managed because of the effects on the phase lag and the amplitude of filtered signals. In Chu et al. [5], a general operational scheme is outlined to define the hardware functions required in a structural control system when choosing the control hardware. This work claims that the major tasks of constructing the code in the digital control system are not only those dealing with calculating the control force and the multiple fail-safe protection functions, but also those needed for conducting other real-time signal manipulations. Gavin [6] relates on the testing and modelling of SA electrorheological fluid devices and their application to seismic control a three degrees of freedom building model on a shaking table. He shows the implementation of the control rule by a circuit including transistors and switches and analyzes all the sources of operational delays of the whole system.

Casciati et al. [7] summarize the advantages and disadvantages of collocated and noncollocated systems as a function of the particular needs of the control system. Suggestions regarding the hardware and software configurations are given, so that they can be used for many different applications in the field of SA control, with only a minimum effort of updating, when needed, due to the evolution of technology.

Finally Gattulli et al. [8] discuss several aspects in the implementation phases of SA protection systems. Among them, the important role of measuring equipment for a good registration of the structural response, the monitoring and control of the state device, and the collection of information and transmission to a digital control system. Furthermore, the necessity of a ground reference to avoid a large amount of noise and the effects of unmodeled dynamics in the measured responses are also investigated.

Therefore, the operation and assessment of the efficacy and efficiency of SA structural control systems based on the adoption of MR devices are still objects of research. For a typical SA control system based on MR dampers to work properly, complex electronic equipment is required. This work attempts to present a comprehensive description and offer suitable solutions for a number of specific aspects that characterise the experimental activity on SA control systems, most of them being atypical for any other kind of structural testing. Authors also highlight undesired and unavoidable effects (delays, control malfunctions, etc.) and provide insights into the way to reduce their incidence.
The above discussion is done with reference to a case study that is a recent experimental campaign, which the authors have been conducting on a full-scale steel structure semiactively controlled via MR dampers. The tests were performed at the Structural Engineering Laboratory of the University of Basilicata in Potenza (Italy) in the framework of the "JET-PACS" program [9] provided by Italian ReLUIS Executive Project.

In the following, Section 2 presents a brief description of the case study, whereas Section 3 provides a deep insight into experimental issues, separately discussed for each of the three components of the SA control chain (acquisition, processing and control, and command). Finally, in Section 4 the main conclusions this work lead to are stated.

\section{Case Study: Semiactive Control of a Steel Structure}

In the next sections the main issues to deal with when implementing an experimental campaign on the SA control of a structural model under dynamic actions are discussed. For each of these, the experience gained in a recent laboratory activity by the authors is exploited to provide information useful to potential users aiming at performing similar experimental investigations. The description of this case study is briefly introduced herein, while the results of the tests are out of scope of the paper (refer to [10] for details).

The case study structure consists of a 2-storey one-bay steel frame (steel type S235) with composite steel-reinforced concrete slabs (Figure 1), $3 \mathrm{~m} \times 4 \mathrm{~m}$ in plan, with a total height of $4.5 \mathrm{~m}$. Four lateral beams (cross section IPE180) are welded to the four corner columns (HEB140) and placed at the first and second (top) floors, whereas four lateral beams (HEB220) and two horizontal braces (HEA160) are installed at the ground floor. Four concrete blocks ( $340 \mathrm{~kg}$ each) have been installed to simulate the presence of additional dead loads and of variable loads. The frame is supported on special sliding 1D guides positioned under the base beams, which allow the frame to move in the longitudinal direction only, thanks to the dynamic actuator mounted at the reaction wall.

Chevron-type braces (HEA100) are mounted along the longitudinal direction, on both longitudinal sides (say West and East) of the frame. Those at the ground floor have been used to install (by means of bolted connections) two MR dampers (Figure 1). Therefore the proposed "smart" bracing system is made up by three parts: an elastic or rigid brace according to the type of gusset plate installed at the top of the brace (flexible or infinitely rigid), a SA time-varying damping MR device, and a control algorithm.

The two prototype MR dampers have been provided by Maurer Söhne (Munich, Germany). The total length of each device is $595 \mathrm{~mm}$ with a stroke of $\pm 25 \mathrm{~mm}$, the external diameter is $100 \mathrm{~mm}$, and the mass is about $16 \mathrm{~kg}$. A maximum force of about $30 \mathrm{kN}$ can be developed along its longitudinal axis, whereas the presence of special spherical pin joints at both ends prevents the rise of bending, shear, and torsional moment in the piston rod. A patented magnetic circuit, composed by 3 coils in series, can generate the magnetic 


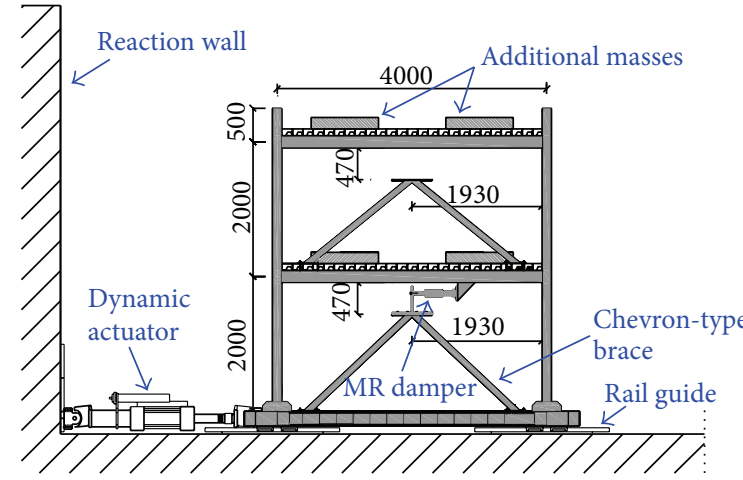

(a)

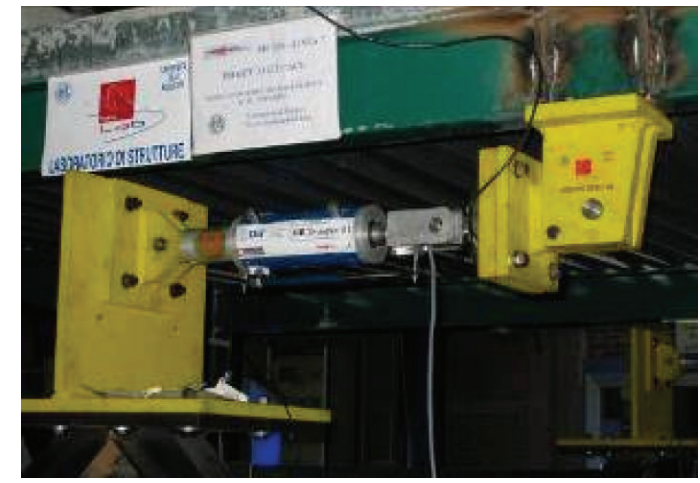

(b)

FIGURE 1: Lateral view of the mock-up structure (a). One of the two MR dampers installed at the first floor (b).

field in the device. The internal circuit of the damper has the following properties: a resistance of $3.34 \Omega$, a reactance of $1.27 \Omega$, an impedance (modulus) of $3.57 \Omega$, and an inductance of $276 \mathrm{mH}$. The current in the circuit is provided in the range $0 \sim 3 \mathrm{~A}$ by a specific power supply.

The overall experimental campaign consisted of 50 tests, different from each other for seismic load imposed via the shaking table facility, control algorithm to drive the MR devices, and maximum intensity of current allowed to be supplied to the dampers. Seven natural records were selected and scaled to be compatible with the response spectrum provided by Eurocode 8 for soil type B, Seismic Zone 1. Four control algorithms have been tested, imposing a maximum level of current in the interval $[1.0,2.5] \mathrm{A}$ with a step size of $0.5 \mathrm{~A}$. A total of 27 acquisition channels were employed to capture the structural response, the most significant being described in Table 1 together with information about the transducers. Special additional electronic equipment, properly addressed to drive the SA tests, has been further employed, as it is presented in the following sections.

\section{Experimental Implementation of a Semiactive Control System: Issues and Possible Remedies}

SA structural control systems are based on the adoption of additional devices able to modify in a short time their stiffness and/or their dissipating capabilities. These properties can be adjusted according to the instantaneous response of the hosting structures (feedback) and/or to the instantaneous properties of the earthquake input (feed-forward). In both cases, the relationships between the observed quantities and the corresponding optimal calibration of the adjustable devices are provided by control algorithms.

A number of scientific papers have subsequently proposed many different ideas to define control algorithms for SA control systems. However, all the proposals can be considered belonging to one of the following families: (i) control algorithms derived from a known control theory applied to provide a full control authority. In this case, SA devices are looked at as-purely reactiveforce actuators and the full control authority has to be clipped in order to match the effective capabilities of the dampers;

(ii) control algorithms based on a sound physical sense, where SA devices are typically seen as smart damping devices for which the amount of dissipation can be quickly regulated.

Control algorithms belonging to the first family typically aim to make the SA device emulate as good as possible the behavior of an actuator driven by an active control strategy to achieve given performance objectives. Recent developments showed how such controller may be effective, for example, because of being addressed to impose performance requirements even over finite frequency ranges [11] or to artificially modify the damped modes of the controlled dynamic system $[12,13]$.

They usually require real time measurements of the full system state. In the case of civil structures, this corresponds to real time evaluation of displacements and velocities at every single significant degree of freedom (DOF). However, direct measurement of displacement and velocity is seldom a viable opportunity: typical dynamic acquisitions on civil structures rely on accelerometric recording. The calculation of displacements and velocities from accelerations can be done either by on line double integration or through the adoption of state observers. In both cases, however, the corresponding numerical computations are associated to the following issues.

(1) Vibration periods of structures prone to earthquake effects are typically in the range $0.1-1.0 \mathrm{~s}$. The bandwidth of a SA damping system must be significantly higher than the dynamics that it intends to control. Therefore, real time modification of the damping properties of SA devices should happen in the range $1-10 \mathrm{~ms}$, including both computation times and mechanical delays $[14,15]$ and, in turn, on line double 
TABLE 1: Acquisition of the structural response for the case study experiments.

\begin{tabular}{lc}
\hline Transducer & Physical quantity acquired \\
\hline $\begin{array}{l}\text { Servoaccelerometers } \\
\text { Columbia, model SA-107LN }( \pm 1 \mathrm{~g})\end{array}$ & Acceleration of the base level \\
FGP, model FA101-A2 $( \pm 2 \mathrm{~g})$ & Acceleration of the 1st floor \\
\hline Temposonic $\pm 250 \mathrm{~mm}$ & Acceleration of the 2nd floor \\
digital transducers & Absolute displacement of the base level \\
$2 \mu \mathrm{m}$ resolution & Absolute displacement of the 1st floor \\
\hline $\begin{array}{l}\text { AEP load cells } \\
\text { model TC4, } 50 \mathrm{kN}\end{array}$ & Absolute displacement of the 2nd floor \\
\hline $\begin{array}{l}\text { Penny and Giles LP } \\
\text { displacement transducers } \\
\text { model HLP190/SA, } \pm 50 \mathrm{~mm}\end{array}$ & MR damper east side axial force \\
\hline
\end{tabular}

integration and/or state observation could take too long to meet this tight schedule.

(2) Top class CPUs able to match such specifications are typically included in computers needing a power source, whose maximum probability of shortage corresponds to moments when they would be more needed (earthquakes, severe storms, etc.).

Controllers belonging to the 2nd family (sound physical sense) are typically simpler and usually require less measurements, which often are made in the close surroundings of the devices. Therefore, the computational effort is fairly moderate and, in principle, it could be sustained by small, battery-powered computing systems. Apart from the initial, bistate, and coefficientless control algorithm suggested by Crosby and Karnopp [16], some other different proposals, based on a clear physical sense, can be found in the scientific literature. Inaudi [17] proposed a control strategy for SA friction dampers, based on the concept that a variable friction coefficient has to be adjusted so as to be proportional to the deformation of the device itself. A modified version of this controller for variable-damping device is shown in [18]. The original idea of Inaudi was subsequently developed in a more complex control algorithm taking into account both relative displacement and velocity of the damper [19]. Stammers and Sireteanu [20], in the context of vehicle suspension design, introduced a control algorithm for SA friction dampers aimed to reduce the accelerations of the main body of a car. They extended their idea to the case of seismic structures in Stammers and Sireteanu [21]. Many authors [22-25] have proposed a resetting control scheme to drive SA dampers. The resetting principle is based on the introduction of a SA bracing system, to be considered as an energy extractor, composed by an elastic element and a damping device. The elastic element is adopted to temporarily store strain energy to be quickly damped out during short dissipation cycles. Resetting systems can be analyzed in the framework of control theory, as shown by Occhiuzzi and Spizzuoco [26]. Other control logics based on physical concepts are shown in [27-31].
The interest of authors in this paper only indirectly involves control algorithms, given that the particular experimental issues to deal with in some way depend on the controller/controllers selected to be implemented and used for the test. However, authors examined the whole control chain that includes

(i) an acquisition phase, where selected parameters of the structural response and/or of the ground acceleration are measured;

(ii) a processing-decision stage, where the measured quantities are processed by the control algorithm leading to decisions about the calibration of the devices;

(iii) a command activity, where decisions made by the algorithm are transferred to the adjustable devices, typically by means of electrical signals.

Experimental activities involving SA devices require special attention in solving several specific aspects that characterize each of the three phases pointed out above. Most of them are uncommon in any other kind of structural testing. In the following the main issues related to the feedback case are highlighted, also describing how they were dealt with for the case study. Undesired and unavoidable effects, together with the possible ways to reduce their incidence, are also discussed.

3.1. Acquisition Phase. When feedback control is adopted, the properties of the variable dampers have to be adjusted according to the instantaneous response of the hosting structures. Therefore selected parameters of the structural response have to be continuously measured during the tests. The number and type of response parameters to be monitored depend on the characteristics of the structure, the number of SA devices installed, the way they are mechanically connected to the hosting structure, and, not least, on the particular control algorithm adopted.

For the case study, four "co-located" control algorithms were adopted. In order to work they needed only a few parameters to be acquired, as shown in Table 2. The structural response instead was evaluated by measuring the quantities 
TABLE 2: Acquisition needed to the control activity.

\begin{tabular}{lc}
\hline Transducer & Physical quantity acquired \\
\hline LVDT & Absolute displacement of the base level \\
LVDT & Absolute displacement of the 1st floor \\
LVDT & Absolute displacement of the 2nd floor \\
- & Voltage commanding the devices \\
Voltage transd. & Voltage in the damper's circuit \\
Current transd. & Current in the damper's circuit \\
LVDT & Relative displacement of the dampers \\
Load cell & Axial force on dampers piston \\
\hline
\end{tabular}

(force in the dampers, floors' accelerations, and displacements) described in Table 1. Unlike other more common experimental tests on structure, this kind of investigation requires the acquisition (and the management) of unusual quantities such as the voltage and the intensity of current inside the installed devices.

Since MR dampers are inductive loads, it is generally needed to stabilize the current loop operating with them. Two means of stabilizing can be generally adopted, when they are not already included in the power supplier: (a) a capacitance in parallel with the output and (b) a series-connected resistor capacitor network in parallel with the output. The value of capacitor or resistor has to be chosen according to the value of the load inductance. For the recent experience herein referred as case study, two operational power suppliers (model BOP 50-4 M from Kepco Inc., New York, USA) were adopted, being able to provide the feeding current to the MR devices, with an output power of $200 \mathrm{~W}$, a maximum input power of $450 \mathrm{~W}$, and an output range of $\pm 50 \mathrm{~V}$. In that case, a $1.0 \mu \mathrm{F}$ capacitance was mounted in parallel to the output with the aim of stabilizing the current loop ("A" in Figure 2).

Other special tools adopted for the acquisition phase of those tests were a 10-to-1 voltage attenuator (" $\mathrm{C}$ " in Figure 2) needed to scale the $\pm 50 \mathrm{~V}$ output signal from one power supply in order to make it readable by the acquisition board, the latter being a National Instruments (NI) PXI 6259 data acquisition board with 16 inputs and 4 outputs $( \pm 10 \mathrm{~V}$ voltage signals, 16 bit resolution and $2800 \mathrm{kHz}$ maximum sampling rate); a NI chassis ("D" in Figure 2); a NI Labview Professional Development System (release 8.5), that is, the environment in which the software needed to acquire and generate all the analog signals involved in the experimental tests has been purposefully written (" $\mathrm{B}$ " in Figure 2 is a video screen); a NI digital multimeter able to measure the intensity of current in the circuit of one damper; and two connector blocks.

Most of the control algorithms continuously calibrate the smart device according to the instantaneous value of the input parameters. Moreover, in many cases the control algorithms drastically change the behavior of the devices when the sign of one of the acquiring input parameters changes. That said, it is crucially important to be able to acquire signals as clean as possible and free from noise or other disturbances that may distort the trend. On the other hand the use of on-line filtering should be strongly limited in this kind of test: the unavoidable delays it causes may be not compatible with the correct, effective working of the control algorithm. Therefore special attention should be paid to the choice of the transducers to be used for the tests (the overall quality and the particular features) and to all the other technical aspects that help to reduce noise on the input signals (type and quality of wires, their connection, and so on).

3.2. Processing and Control Logic Phase. In this stage the measured quantities are processed by the control algorithm leading to decisions about the calibration of the devices. To make this phase effectively work, the following is needed:

(i) to use high performance hardware, able to work in real time, as much as possible; that is, a high speed $\mathrm{CPU}$ is certainly strongly recommended; the aim is to reduce the processing time, therefore to reduce the response time of the control system;

(ii) to adopt an operative system properly designed to work in real time, together with an advanced development system dedicated to create applications for test, measurement, and control (Figure 3);

(iii) to create a stand-alone executable able to reproduce the logic on which the control algorithm is based; this program should be organized in the most easy and rational manner in order to reduce the processing time within the strictly necessary amount.

Control malfunctions may happen during the tests. Depending on many factors, it is possible that in certain instants of the test the electronic system described above is not able to take the right decision (i.e., the one that would derive from the correct application of the control logic) nor to turn it in the right command to the power supplier. If the control system is properly designed, these failures have a low incidence (i.e., occur only in a few instants of the motion) and can be generally neglected.

For the case study, four controllers were adopted. They were "co-located" since they are able to command a change in real time of the dynamical properties of the dampers according to the actual values of measured quantities (displacements, velocities, and forces) in the close surroundings of the dampers, not accounting for the complete state measurement of the whole structures. The algorithms are briefly referred to as "Energy" (aiming to maximize the energy extracted by the dampers from the structure [26]), "Acceleration reduction" (aiming to minimize the amount of absolute accelerations in the main structure and, therefore, to minimize the energy transmission from the ground motion to the structure through the SA brace; based on a proposal of [20]), "Sky hook" (aiming to make the SA damper mimic a sky-hook damper, i.e., a damper constrained to the fixed space [4]), and "Modulated homogeneous friction" (originally proposed by [17], it modulates the current according to the actual response of the structure in terms of floor displacement measured using the so called "prior-to-peak" $P(t)$ operator related to the interstorey drift of the first level). Equations (1)(4), respectively, show how the above controllers have been specialized to be applied to the case study (refer to Figure 4 for symbols). The gain constant $g$ for the last algorithm has 


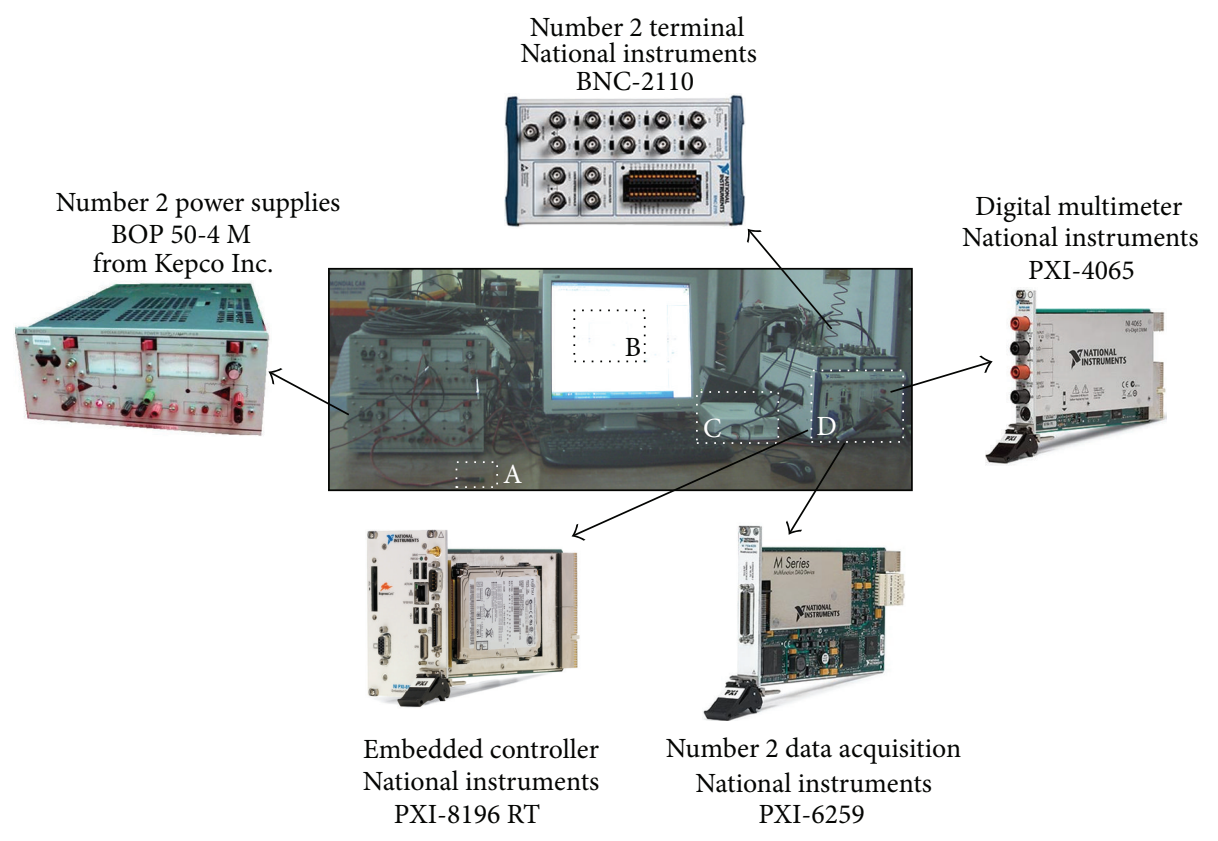

FIGURE 2: Electronic equipment for acquisition and control during the JET-PACS experimental tests.

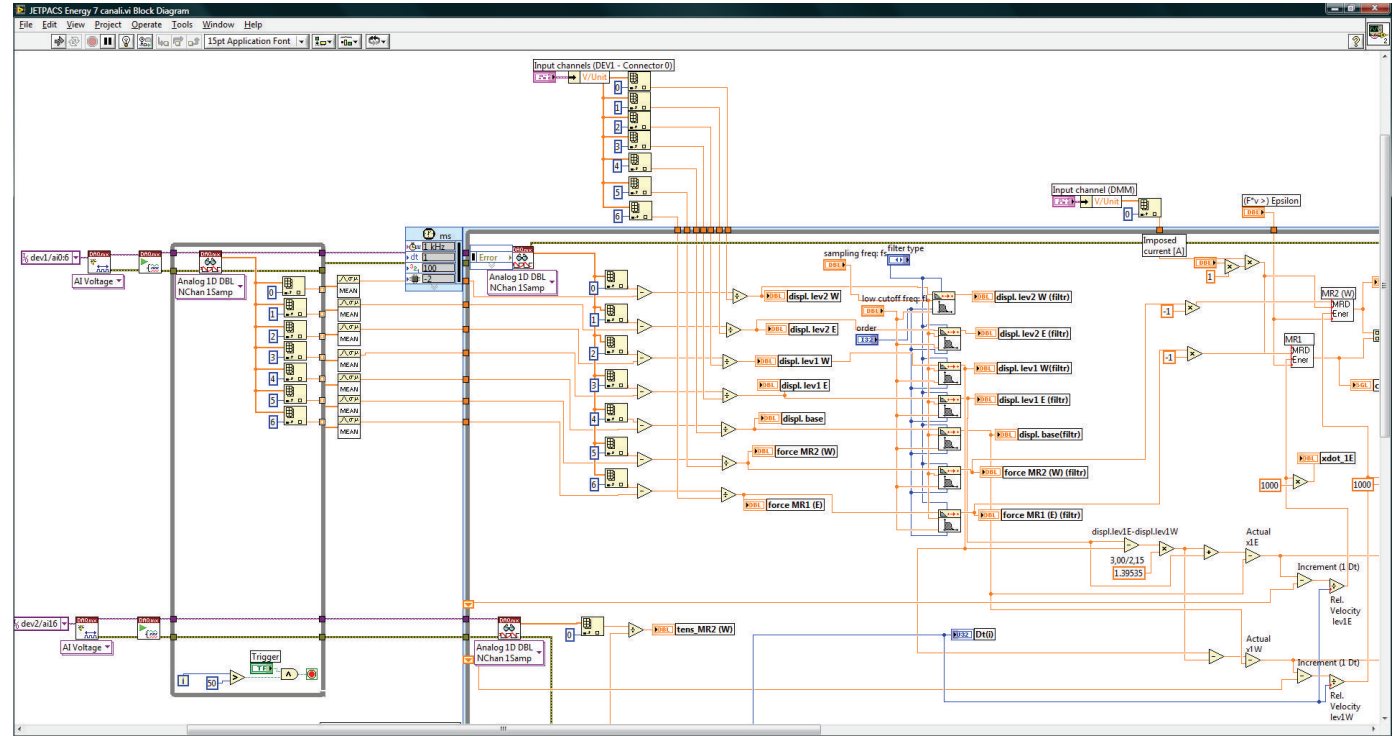

FIGURE 3: One of the programs written for the case study experience: a labview video screen.

been assumed so as to give the maximum intensity of current to the damper when the 1st interstorey drift ratio achieves the value of 0.005 (i.e., $x_{f}-x_{g}=10 \mathrm{~mm}$ ):

$$
\begin{gathered}
\text { if } F_{b}(t) \cdot \dot{x}_{f}(t)>0 \quad \text { then } i(t)=i_{\max }, \\
\text { if } F_{b}(t) \cdot \dot{x}_{f}(t)<0 \text { then } i(t)=i_{\text {min }}=0, \\
\text { if } x_{f}(t) \cdot\left\lfloor\dot{x}_{f}(t)-\dot{x}_{b}(t)\right]>0 \text { then } i(t)=i_{\min }=0, \\
\text { if } x_{f}(t) \cdot\left[\dot{x}_{f}(t)-\dot{x}_{b}(t)\right]<0 \quad \text { then } i(t)=i_{\max },
\end{gathered}
$$

$$
\begin{gathered}
\text { if } \frac{\dot{x}_{f}(t)+\dot{x}_{g}(t)}{\dot{x}_{f}(t)}<0 \quad \text { then } i(t)=i_{\text {min }}=0, \\
\text { if } \frac{\dot{x}_{f}(t)+\dot{x}_{g}(t)}{\dot{x}_{f}(t)}>0 \quad \text { then } i(t)=i_{\max }, \\
i(t)=g \cdot|P(t)| \quad \text { with } g=0.1 * i_{\max } \mathrm{A} / \mathrm{mm} .
\end{gathered}
$$

The effectiveness of each controller in reducing the seismic demand to the steel structures is discussed by Caterino et al. [10] and is out of the scope of the present paper. Rather herein it is worth highlighting, among the experimental 


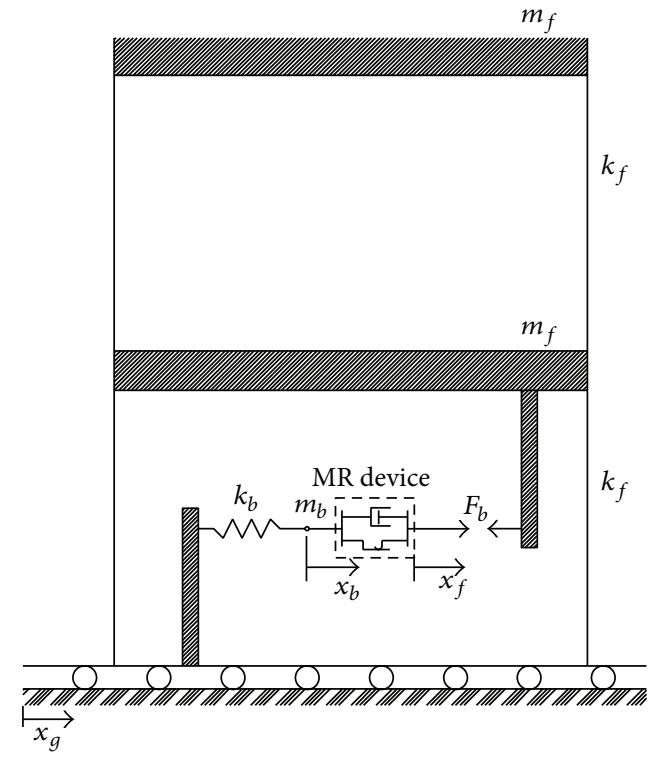

Figure 4: Model of the tested structure.

issues emerging for such application, the aspects related to control malfunctions introduced above, the latter resulting to be controller-dependent.

For each test, all the time instants corresponding to a wrong operation of the control system have been singled out, by checking when the command signal to the devices did not provide the control required by the analytical formulation of the algorithm. Then, the duration of the strong ground motion has been evaluated for each applied seismic input as the time interval between the first and the last peak acceleration over $0.2 \mathrm{~g}$ for the heavier earthquakes and over $0.1 \mathrm{~g}$ for the other ones, and the number of wrong operations falling in the strong motion time interval has been simply derived from the total number of failures.

Table 3 reports the maximum value, computed on all the tests performed by applying the same control algorithm, of the percentage of undesired working for the whole duration of the test as well as for the duration of strong motion only. The table makes clear that malfunctions assumed a quite significant incidence only for the tests driven according to the Energy control logic, while they resulted to be almost null in the other tests. All the adopted algorithms are such that they change the command signal each time a given function of the time changes its sign, that is, it passes from zero. Being the equipment was the same in all tests, only the Energy algorithm requires measurement of forces in addition to measurement of displacements (1). The higher incidence of malfunctions on the tests driven by the Energy algorithm, in comparison to the other controllers, could be motivated noting that only Energy uses, as reference for deciding the command signal, the product of two quantities, that is, force and velocities, different in nature and measured by transducers based on quite different physical principles and manufactured with different technologies. Processing such different signals causes unexpected and unpredictable "black-outs" on data processing.
TABLE 3: JET-PACS: percentage malfunctions of the control system driven by different algorithms.

\begin{tabular}{lcc}
\hline Controller & Failures & During strong motion \\
\hline Energy & $6.48 \%$ & $3.33 \%$ \\
MHF & $0.02 \%$ & $0.03 \%$ \\
Sky Hook & $0.03 \%$ & $0.03 \%$ \\
Acc. red. & $0.06 \%$ & $0.02 \%$ \\
\hline
\end{tabular}

Further practical factors that might have deleterious impact on the performance of the control algorithm are worth describing. Major problems derive from unmodeled nonlinear dynamics and nonstationary phenomena. Nonlinearity occurs naturally in most damping devices when subjected to severe loads. This is as a consequence of the damper internal force exceeding the damping material yield point, that is, exhibiting hysteresis [32]. For the case of SA fluidbased dampers, an additional source of nonlinearity comes from the damper plunger seals. They are designed to maintain alignment and stop the fluid from leaking. Still, in practice, they build up significant static friction that restricts the range of velocities where the damper moves. For the large SA dampers used in Civil Engineering applications, the static friction force is usually high and needs to be overcome before the control system is unlocked.

Another important issue is related with the damper mounting and its alignment. Ideally, forces are transferred to the damper through perfect joints. However, in real scenarios mountings are likely to allow for some clearance in the damper connection. This causes the well-known backlash effect and is responsible for a loss of motion when the movement is reversed.

Additionally, two nonstationary phenomena are important when considering the damper performance within a reasonably long time window. Firstly, after a period of inactivity, MR dampers can exhibit a singular start-up phase in which they provide reduced forces. Some MR dampers need a number of cycles before settling and delivering a uniform response and their distinctive hysteretic loop [33]. This is due to an initial nonhomogeneous distribution of the microparticles inside the MR fluid. A second aspect is associated with the increment of the temperature when MR dampers are forced to work for long periods of time under highly demanding operational ranges. This can be the case, for instance, of dampers fitted into long-span bridges under intense storms.

Authors recall that the above issues can reduce the effectiveness of the SA control system, and hence, they need to be compensated for. Simple control algorithms that do not consider those effects are likely to perform poorly in realistic scenarios. For instance, they could fail in providing an appropriate command signal upon an earthquake strike. Thus, robust controllers that fully embrace the aforementioned nonlinear and nonstationary features are needed in order to enable most efficient, reliable, and safe SA control systems.

3.3. Command Delivering Phase. In this step, decisions made by the algorithm are transferred to the SA devices typically by 


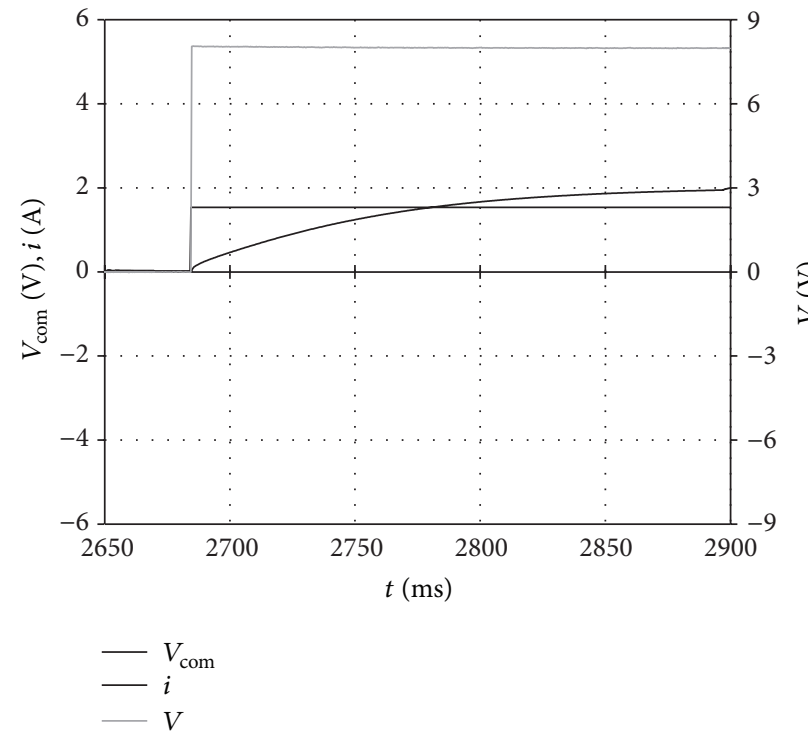

(a)

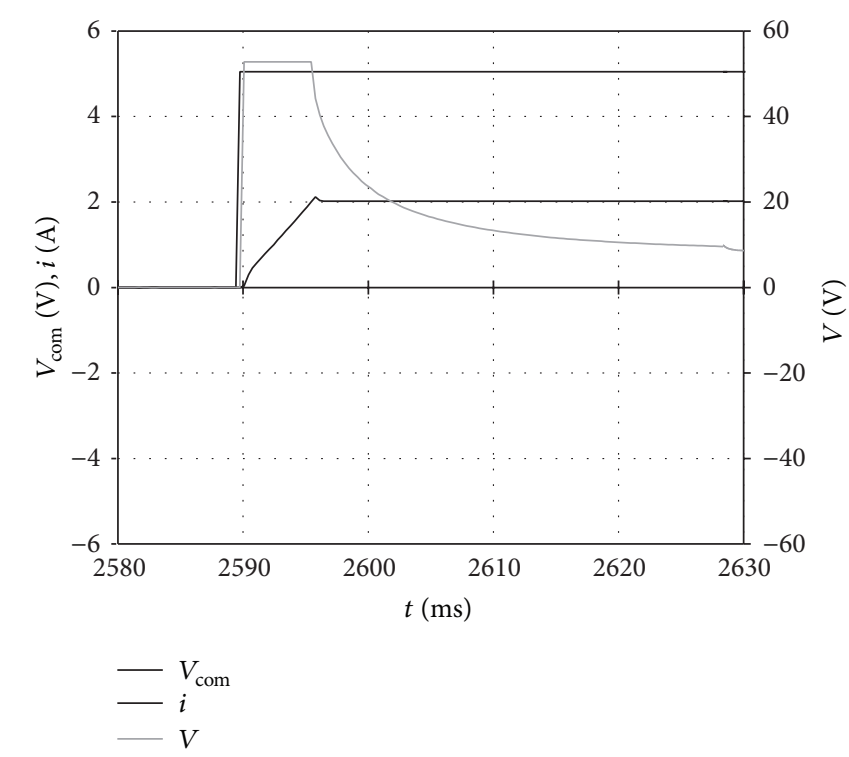

(b)

FIGURE 5: MR damper's electrical behaviour when a "switch on" $0 \rightarrow 2 \mathrm{~A}$ is imposed using a voltage-driven (a) or a current driven (b) approach.

means of electrical signals. This happens thanks to a voltage command signal generated in output from the computer and directed in input to the suppliers. Once the command signal is received, these have to generate a new signal in output addressed to modify the current inside the dampers (generally a power supplier is needed for each installed device). These operations follow each other continuously during the entire test and should be as fast as possible. Actually, generally speaking, response delays of the control system may have a significant incidence on the effectiveness of the SA control strategy.

To drive the mechanical behaviour of the SA dampers during the test, according to decision of the controller, it is possible to assume a current-driven or a voltage-driven to control the intensity of the current inside the damper. Advanced power suppliers (referred to as "power sourcepower sink") allow to choose indifferently one of the two different schemes. Depending on this choice, the promptness of the dampers strongly changes. In a voltage-driven scheme, the power supplier provides a fixed voltage and the current slowly modifies until it reaches a value corresponding to the ratio voltage/resistance. In current-driven operations, the power supplier provides a fast changing voltage spike so as to quickly modify the current inside the damper. If the current must be increased, the power supply provides for a short period a voltage spike and then sets the voltage to the reference value, whereas if the current must be decreased, a negative spike of voltage is issued. The main differences of the two different schemes are graphically shown in Figure 5 based on the experimental activity of Caterino et al. [15]. Both diagrams correspond to a "switch on" $(0 \mathrm{~A} \rightarrow 2 \mathrm{~A})$ of the damper. Figure 5(a) refers to the voltage driven scheme, whereas (b) is referred to a test made using a current driven approach. From Figure 5(a) it can be observed that when the command signal $V_{\text {com }}$ given in input to the supplier goes from 0 to $1.54 \mathrm{~V}$, the voltage $V$ given by the power supplier to the damper instantaneously varies from 0 to $8.25 \mathrm{~V}$, whereas the current $i$ slowly increases from 0 to $2 \mathrm{~A}$, due to the electric inductance of the coils inside the damper, taking about $215 \mathrm{~ms}$ to reach the desired value.

Figure 5(b) shows that when $V_{\text {com }}$ goes from 0 to $5 \mathrm{~V}$, after about $1 \mathrm{~ms}$ the voltage sent by the power supplier to the damper reaches a spike of about $50 \mathrm{~V}$ which lasts about $6 \mathrm{~ms}$, that is, the time needed by the current to increase from zero to desired value. After this time, the voltage generated by the power supply falls down to the steady-state value of $8.25 \mathrm{~V}$. Finally, since the viscosity of the MR fluid is related to the current intensity that generate the magnetic field, rather than to the voltage, adopting a current-driven approach should be always considered as mandatory to bound the response time within few milliseconds and, in turn, to do not appreciably affect the effectiveness of the overall control system. It is worth noting that in voltage driven operations the power requirement is about $16 \mathrm{~W}$ which becomes about $100 \mathrm{~W}$ in current driven operations.

As a matter of fact, the response delay of a SA control system does not uniquely depend on the above considerations, since it can be considered as the sum of these four terms (Figure 6):

$$
\tau=\tau_{a}+\tau_{c}+\tau_{e}+\tau_{m}
$$

where

$\tau_{a}$ is the time delay of the control electronics which includes, consecutively, the time intervals associated to signal acquisition and processing through the algorithm;

$\tau_{c}$ is associated to the power supply, being the time interval starting when the driving signal (in output 


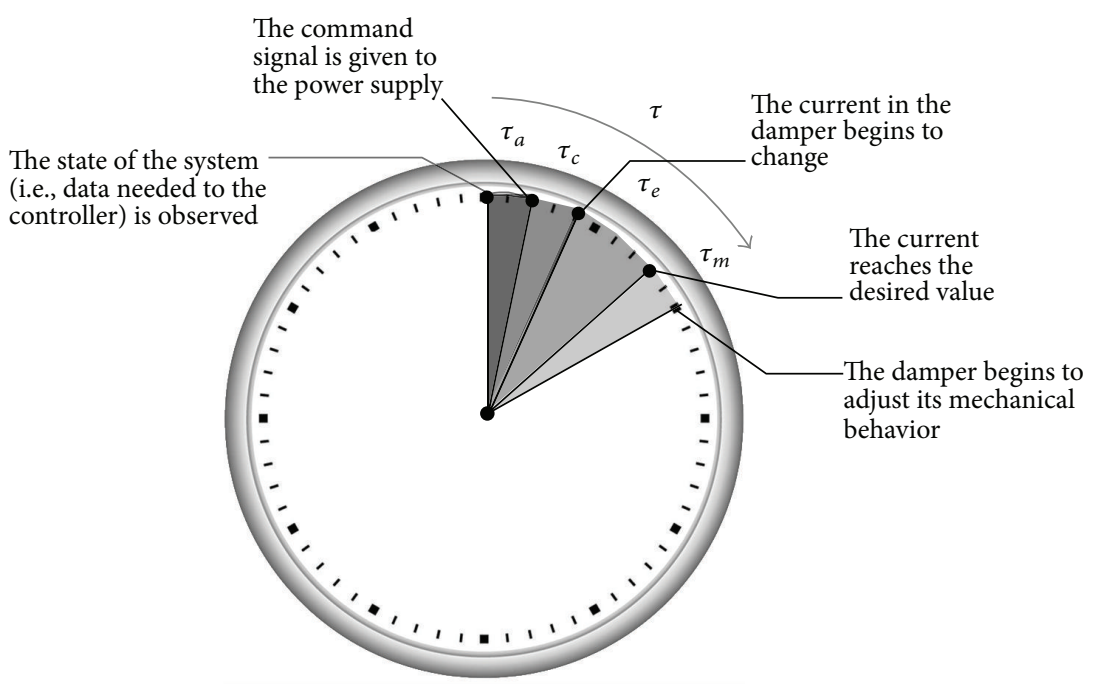

FIGURE 6: Schematic representation of time delays in a control chain involving MR dampers.

from the algorithm and in input to the power supply) is issued and ending when the current (in output from the power supply and in input to the device) begins to change;

$\tau_{e}$ is the time delay of the electrical part of the damper, which is the time interval starting when the current (in input to the device) begins to change and ending when the current reaches the commanded nominal value within a $\pm 5 \%$ tolerance;

$\tau_{m}$ is related to the mechanical part of the damper, representing the time interval between the instant when the current (in input to the device) reaches its nominal value and the instant when the damper begins to react, that is, begins to adjust its mechanical behaviour.

For the SA control system herein assumed as case study, the authors measured, in different conditions, the amount of each of the above delay fractions, concluding that time delays $\tau_{a}$ and $\tau_{c}$ turned out to assume a rather stable value equal to $0.4 \mathrm{~ms}$ for both on $\rightarrow$ off and the off $\rightarrow$ on commands, resulting to be practically independent from the maximum commanded current. The mechanical response time $\tau_{m}$, measured in both the switch off and switch on operations, resulted in an almost stable value, on average equal to $1 \mathrm{~ms}$. Finally electrical delays $\tau_{e}$ resulted to be the most part of $\tau$, strongly dependent on the level of current intensity as well as on the scheme (voltage- or current-driven) adopted, according to what is said above. When the currentdriven operations have been imposed, $\tau_{e}$ turned out to be in the interval $2 \sim 6 \mathrm{~ms}$ for the on $\rightarrow$ off switch and $2 \sim 9 \mathrm{~ms}$ for the off $\rightarrow$ on phases. Figure 7 shows an example of such evaluation, in the case of a switch on $(0 \rightarrow 2 \mathrm{~A})$ commanded by the controller when the velocity between the damper's ends changes in the sign.

Analyzing and linearly interpolating all the data registered for the $\tau_{e}$ delay within the above campaign allowed us to define the following formulas valid for the current-driven

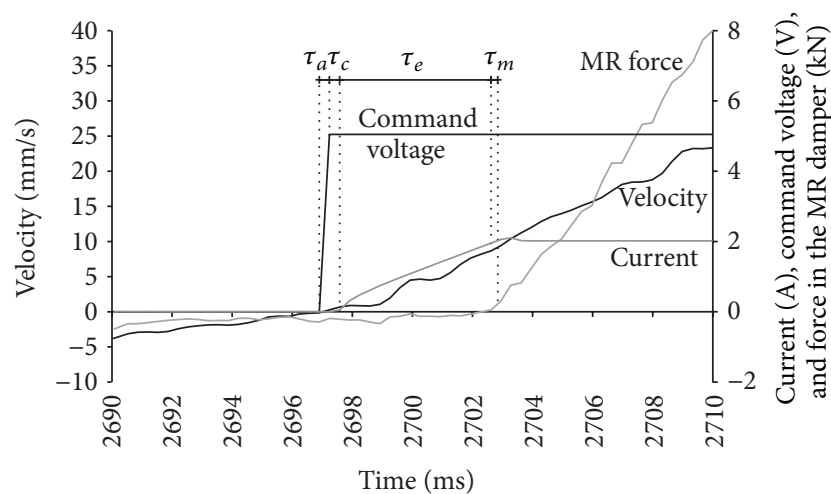

FIgURE 7: Evaluation of the response time of the case study SA control system under an off $\rightarrow$ on command.

scheme ( $i$ is the intensity of current), respectively, for the cases of switch on and switch off of the smart device. The statistical analysis of all the observed delays also leaded to define a Gaussian distribution of the electrical delay normalized by the intensity of the current, with mean value and standard deviation, respectively, equal to $2.478 \mathrm{~ms} / \mathrm{A}$ and $0.339 \mathrm{~ms} / \mathrm{A}$ for the case of switch on, equal, respectively, to $1.786 \mathrm{~ms} / \mathrm{A}$ and $0.303 \mathrm{~ms} / \mathrm{A}$ for the case of switch off:

$\tau_{e}=3.0 i-0.8>0$ Switch on command, 0 to $i$ Ampere,

$\tau_{e}=1.4 i-0.6>0 \quad$ Switch off command, $i$ to 0 Ampere.

The above expressions and distributions can be helpful to predict the amount of delays associated to the SA control of a given structure equipped with the examined MR dampers, taking into account the probability that they actually may occur during a seismic excitation. They can also be useful to be involved in the compensation schemes; some SA control algorithms include aiming at reducing the harmful 
effects these unavoidable delays may cause on the control effectiveness in terms of structural response reduction.

Generally speaking, time delays should be taken into account during a laboratory test as well as for a practical application. Many methods of time delay compensation have been developed and experimentally tested; for instance, Symans and Constantinou [34] used an approach to account for time delays based on the prediction of the response with an advance equal to the amount of expected delays, experimentally measured. They introduced a harmonic time delay compensation method that is a simplified approach based on the assumption that the structure responds as an undamped system in free vibration during the time interval between measuring the response and applying the control force. Dong et al. [35], with reference to suspension systems based on the use of MR dampers, proposed a neural network model to compensate for the uncertain time delay of the variable devices. Lee and Kawashima [36], with reference to the SA control of nonlinear isolated bridges dampers, formulated a compensation method for time-delayed systems based on the Newmark's method, also comparing its effectiveness with two above cited harmonic compensation method and the recursive response compensation method [37]. The latter, for the ideal linear system, considers a recursive relationship between the state response and the time-delayed state response obtained by integrating the closed loop system without ground motion.

A recent study [38] conducted a numerical investigation on the case study structure discussed herein, considering two different kinds of delays: a constant transport delay due to the control chain (acquisition-processing-command) and a delay due to the damper's mechanical/rheological time response modelled by means of a first-order filter. A large number of numerical analyses were carried out by applying a significant set of seismic excitations. In most simulations authors showed that the time delays did not cause a significant variation of the controlled structural response. This result is of some interest although it cannot be generalized.

\section{Conclusions}

The authors described part of the lesson they learnt during a recent experimental campaign on a full scale steel structure semiactively controlled via MR dampers. With reference to that case, practical examples of feasible ways to address problematic situations likely to happen in real applications of SA control systems were given.

Experimental investigation of semiactively controlled structures require special attention in managing and solving many specific issues, most of them being strongly atypical for any other type of structural testing. All these aspects were highlighted, grouping them according to the specific control phase (acquisition, processing, and command) they are involved in.

Undesired effects like malfunctions of the electronic control procedure and time delays were discussed. As the latter is concerned, it was showed that using a current-driven scheme rather than a voltage-driven one may help to reduce the damper response delays within few milliseconds, really a short time, in most case negligible, for example, if compared to typical periods of vibration of civil structures.

The promptness of SA MR devices turned out to be almost exclusively dependent on the effectiveness of the electric part of the control hardware. Compared to the operating delays of voltage-driven control schemes, rheology and nature of MR fluids seem to play a negligible role on the response time of SA MR devices, whose adoption requires a careful design of the electric part and of the power supply. In particular, operating a SA MR damper in real time requires a power supply with power source-power sink capabilities. The adoption of current-driven operations is then mandatory for real applications. In addition, control algorithms whose output is expressed in terms of voltage should be avoided, because they hide the additional delay needed by the electrical circuits to reach the desired value of current and, in turn, the desired intensity of the magnetic field.

Authors addressed the work so as to provide information useful to potential users aiming at performing similar, not so common, experimental investigations.

\section{Conflict of Interests}

The authors declare that there is no conflict of interests regarding the publication of this paper.

\section{Acknowledgments}

The work behind this paper has been financially supported by the consortium Reluis with a grant by the Protezione Civile (Italian Emergency Agency). The MR dampers considered in this paper were designed, manufactured, and provided for free by Maurer Sohne (Munich, Germany). The support of both is gratefully acknowledged.

\section{References}

[1] S. Guo, S. Yang, and C. Pan, "Dynamical modeling of magnetorheological damper behaviors," Journal of Intelligent Material Systems and Structures, vol. 17, no. 1, pp. 13-14.

[2] N. Caterino, M. Spizzuoco, and A. Occhiuzzi, "Understanding and modelling the physical behaviour of magnetorheological dampers for seismic structural control," Smart Matererials and Structures, vol. 20, Article ID 065013, 2011.

[3] J. Lozoya-Santos, R. Morales-Menendez, R. A. RamirezMendoza, and C. A. Vivas-Lopez, "Influence of MR damper modeling on vehicle dynamics," Smart Materials and Structures, vol. 22, no. 12, Article ID 125031, 2013.

[4] A. Preumont, Vibration Control of Active Structures, Kluwer Academic, New York, NY, USA, 2002.

[5] S. Y. Chu, T. T. Soong, A. M. Reinhorn, R. J. Helgeson, and M. A. Riley, "Integration issues in implementation of structural control systems," Journal of Structural Control, vol. 9, no. 1, pp. 31-58, 2002.

[6] H. Gavin, "Implementation and modeling of a semi-active control system," in Proceedings of the ASCE Conference on Condition Monitoring of Materials and Structures, pp. 202-217. 
[7] F. Casciati, G. Magonette, and F. Marazzi, Technology of Semiactive Devices and Applications in Vibration Mitigation, John Wiley \& Sons, New York, NY, USA, 2006.

[8] V. Gattulli, M. Lepidi, and F. Potenza, "Seismic protection of frame structures via semi-active control: Modeling and implementation issues," Earthquake Engineering and Engineering Vibration, vol. 8, no. 4, pp. 627-645, 2010.

[9] F. C. Ponzo, A. Di Cesare, C. Moroni et al., "JET-PACS: joint experimental testing on passive and semiactive control systems," L'Ingegneria Sismica in Italia-XIII Convegno Nazionale dell'ANIDIS, Bologna, Italy, 2009.

[10] N. Caterino, M. Spizzuoco, and A. Occhiuzzi, "Shaking table testing of a steel frame structure equipped with semi-active MR dampers: comparison of control algorithms," Smart Structures and Systems. In press.

[11] W. Sun, H. Gao Sr., and O. Kaynak, "Finite frequency $H_{\infty}$ control for vehicle active suspension systems," IEEE Transactions on Control Systems Technology, vol. 19, no. 2, pp. 416-422, 2011.

[12] N. Caterino, "Semi-active control of a wind turbine via magnetorheological dampers," Journal of Vibration and Control. In press.

[13] N. Caterino, C. T. Georgakis, F. Trinchillo, and A. Occhiuzzi, "A semi-active control system for wind turbines," in Wind Turbine Control and Monitoring, N. Luo, Y. Vidal, and L. Acho, Eds., Springer, Berlin, Germany, 2014.

[14] A. Occhiuzzi, M. Spizzuoco, and G. Serino, "Experimental analysis of magnetorheological dampers for structural control," Smart Materials and Structures, vol. 12, no. 5, pp. 703-711, 2003.

[15] N. Caterino, M. Spizzuoco, and A. Occhiuzzi, "Promptness and dissipative capability of MR dampers: experimental investigations," Structural Control and Health Monitoring, vol. 20, no. 12, pp. 1424-1440, 2013.

[16] M. J. Crosby and D. C. . Karnopp, “The active damper," The Shock and Vibrations Bulletin 43, Naval Research Laboratory, Washington, DC, USA, 1973.

[17] J. A. Inaudi, "Modulated homogeneous friction: a semiactive damping strategy," Earthquake Engineering and Structural Dynamics, vol. 26, no. 3, pp. 361-376, 1997.

[18] J. A. Inaudi, "Performance of variable-damping systems: theoretical analysis and simulation," in Structural Control for Civil and Infrastructure Engineering, F. Casciati and G. Magonette, Eds., pp. 301-316, World Scientific, River Edge, NJ, USA, 2000.

[19] Y. L. Xu and B. Chen, "Integrated vibration control and health monitoring of building structures using semi-active friction dampers. Part 1: methodology," Engineering Structures, vol. 30, no. 7, pp. 1789-1801, 2008.

[20] C. W. Stammers and T. Sireteanu, "Vibration control of machines by use of semi-active dry friction damping," Journal of Sound and Vibration, vol. 209, no. 4, pp. 671-684, 1998.

[21] C. W. Stammers and T. Sireteanu, "Control of building seismic response by means of three semi-active friction dampers," Journal of Sound and Vibration, vol. 237, no. 5, pp. 745-759, 2000.

[22] J. N. Yang, J. H. Kim, and A. K. Agrawal, "Resetting semiactive stiffness damper for seismic response control," Journal of Structural Engineering ASCE, vol. 126, no. 12, pp. 1427-1433, 2000.

[23] J. N. Yang and A. K. Agrawal, "Semi-active hybrid control systems for nonlinear buildings against near-field earthquakes," Engineering Structures, vol. 24, no. 3, pp. 271-280, 2002.

[24] L. R. Barroso, J. G. Chase, and S. Hunt, "Resettable smart dampers for multi-level seismic hazard mitigation of steel moment frames," Journal of Structural Control, vol. 10, no. 1, pp. 41-58, 2003.

[25] J. Erramouspe, P. D. Kiousis, R. Christenson, and T. Vincent, "A resetting stiffness dynamic controller and its bench-scale implementation," Engineering Structures, vol. 29, no. 10, pp. 2602-2610, 2007.

[26] A. Occhiuzzi and M. Spizzuoco, "Experimental analysis of a semi-actively controlled steel building," Structural Engineering and Mechanics, vol. 19, no. 6, pp. 721-747, 2005.

[27] H. Sodeyama, K. Suzuki, and K. Sunakoda, "Development of large capacity semi-active seismic damper using magnetorheological fluid," Journal of Pressure Vessel Technology, vol. 126, no. 1, pp. 105-109, 2004.

[28] H. J. Jung, B. F. Spencer Jr., Y. Q. Ni, and I. W. Lee, "State-ofthe-art of semiactive control systems using MR fluid dampers in civil engineering applications," Structural Engineering and Mechanics, vol. 17, no. 3-4, pp. 493-526, 2004.

[29] H. J. Jung, J. E. Jang, K. M. Choi, and H. J. Lee, "MR fluid damper-based smart damping systems for long steel stay cable under wind load," Smart Structures and Systems, vol. 4, no. 5, pp. 697-710, 2008.

[30] F. Weber, H. Distl, G. Feltrin, and M. Motavalli, "Cycle energy control of magnetorheological dampers on cables," Smart Materials and Structures, vol. 18, no. 1, Article ID 015005, 2009.

[31] S. Laflamme, D. Taylor, M. A. Maane, and J. J. Connor, "Modified friction device for control of large-scale systems," in Structural Control and Health Monitoring, vol. 19, no. 4, pp. 548564, 2011.

[32] F. Ikhouane and S. Dyke, "Modeling and identification of a shear mode magnetorheological damper," Smart Materials and Structures, vol. 16, no. 3, pp. 605-616, 2007.

[33] N. Caterino, B. M. Azmoodeh, and A. Occhiuzzi, "Medium to long term behavior of MR dampers for structural control," Smart Materials and Structures. In press.

[34] M. D. Symans and M. C. Constantinou, "Seismic testing of a building structure with a semi-active fluid damper control system," Earthquake Engineering \& Structural Dynamics, vol. 26, no. 7, pp. 759-777, 1997.

[35] X. Dong, M. Yu, Z. Li, C. Liao, and W. Chen, "Neural network compensation of semi-active control for magneto-rheological suspension with time delay uncertainty," Smart Materials and Structures, vol. 18, no. 1, Article ID 015014, 2009.

[36] T. Y. Lee and K. Kawashima, "Semiactive control of nonlinear isolated bridges with time delay," Journal of Structural Engineering, vol. 133, no. 2, pp. 235-241, 2007.

[37] A. K. Agrawal and J. N. Yang, "Compensation of time-delay for control civil engineering structures," Earthquake Engineering \& Structural Dynamics, vol. 29, no. 1, pp. 37-62, 2000.

[38] J. M. Londoño, G. Serino, M. Spizzuoco, N. Caterino, and A. Occhiuzzi, "Effects of MR damper time delay on the structural control effectiveness under seismic loads," in Proceedings of the 14th European Conference on Earthquake Engineering, Ohrid, Macedonia, 2010. 

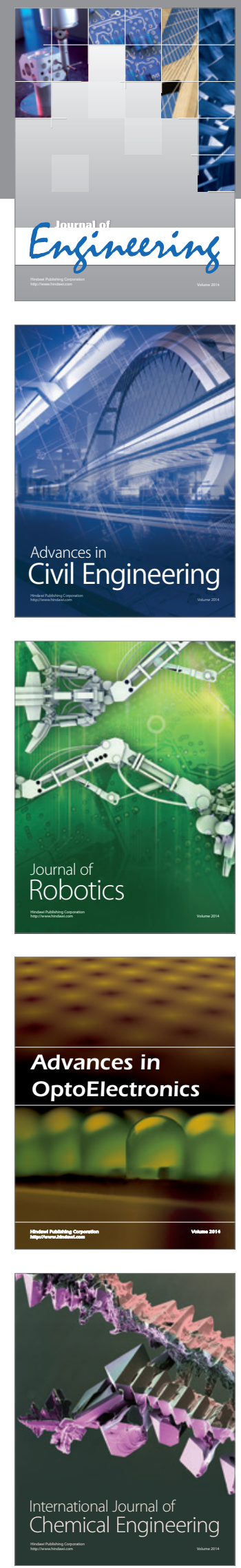

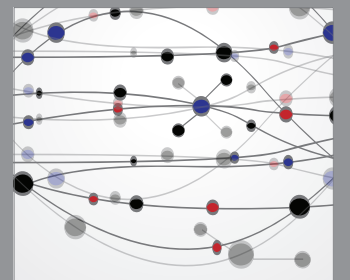

The Scientific World Journal
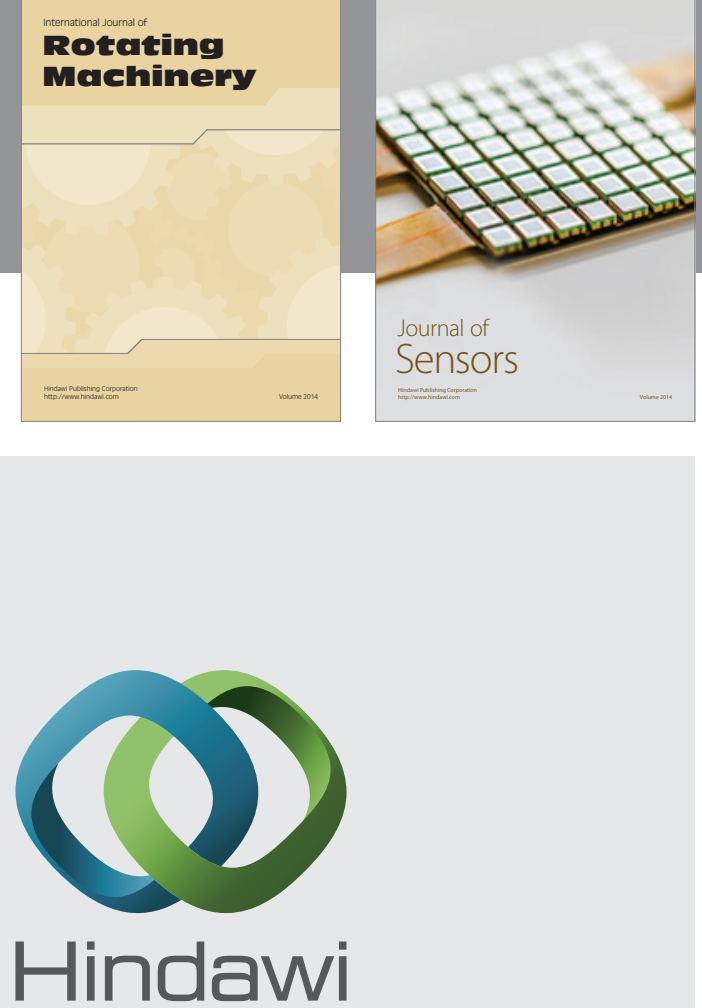

Submit your manuscripts at http://www.hindawi.com
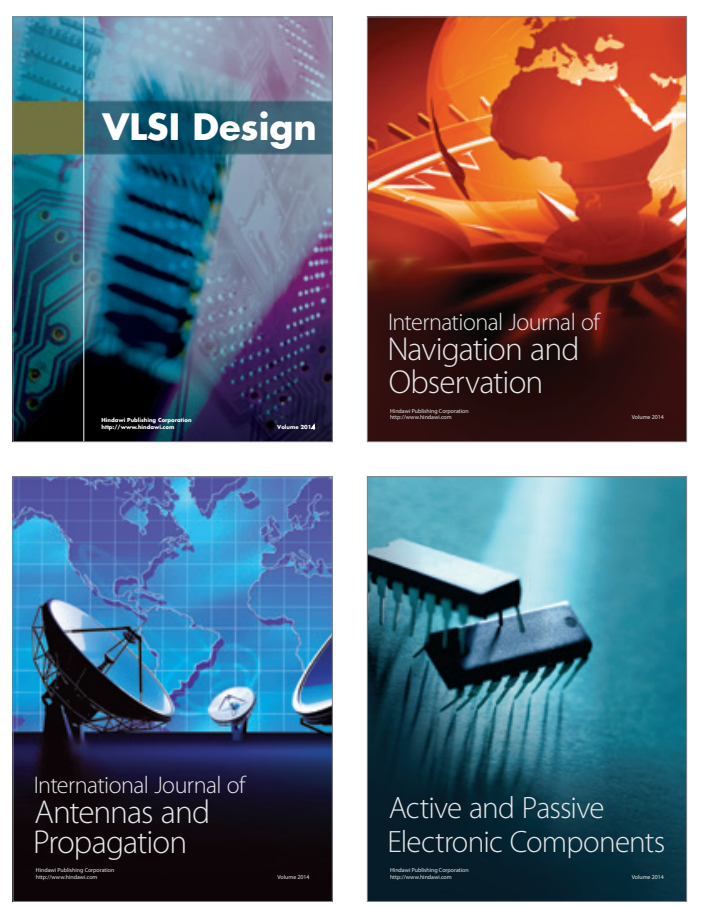
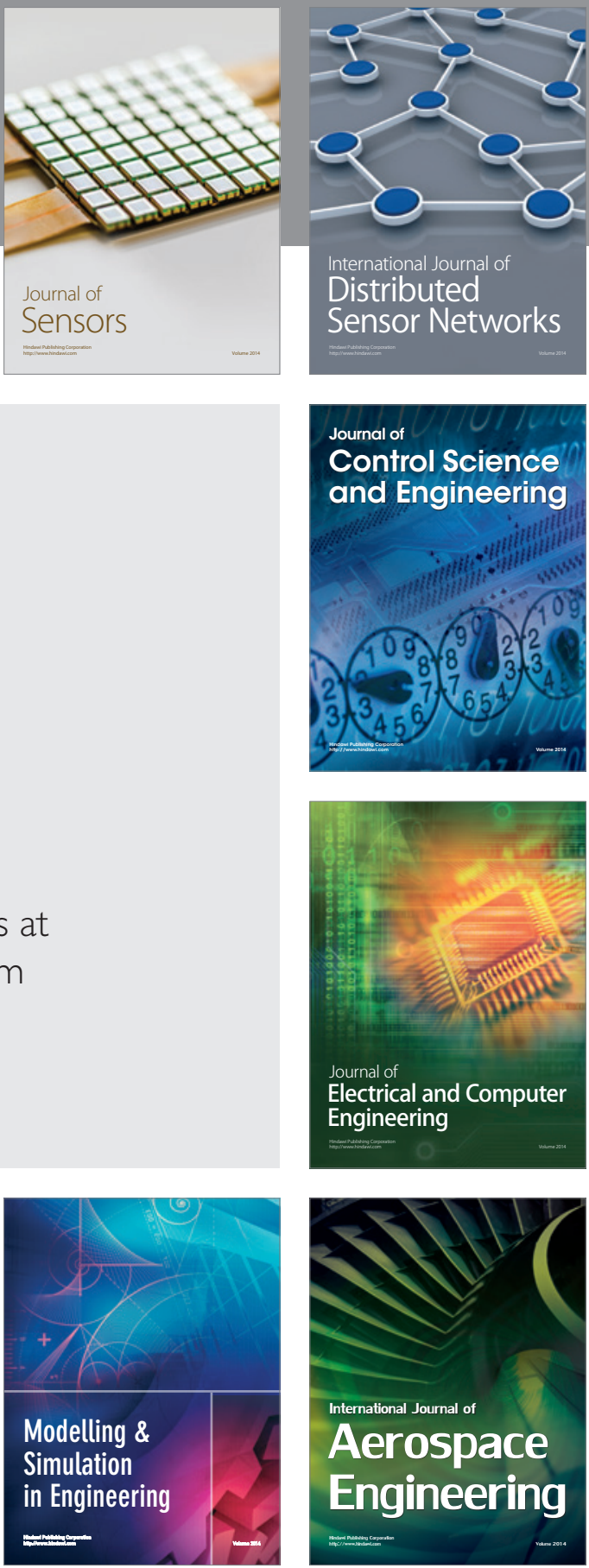

Journal of

Control Science

and Engineering
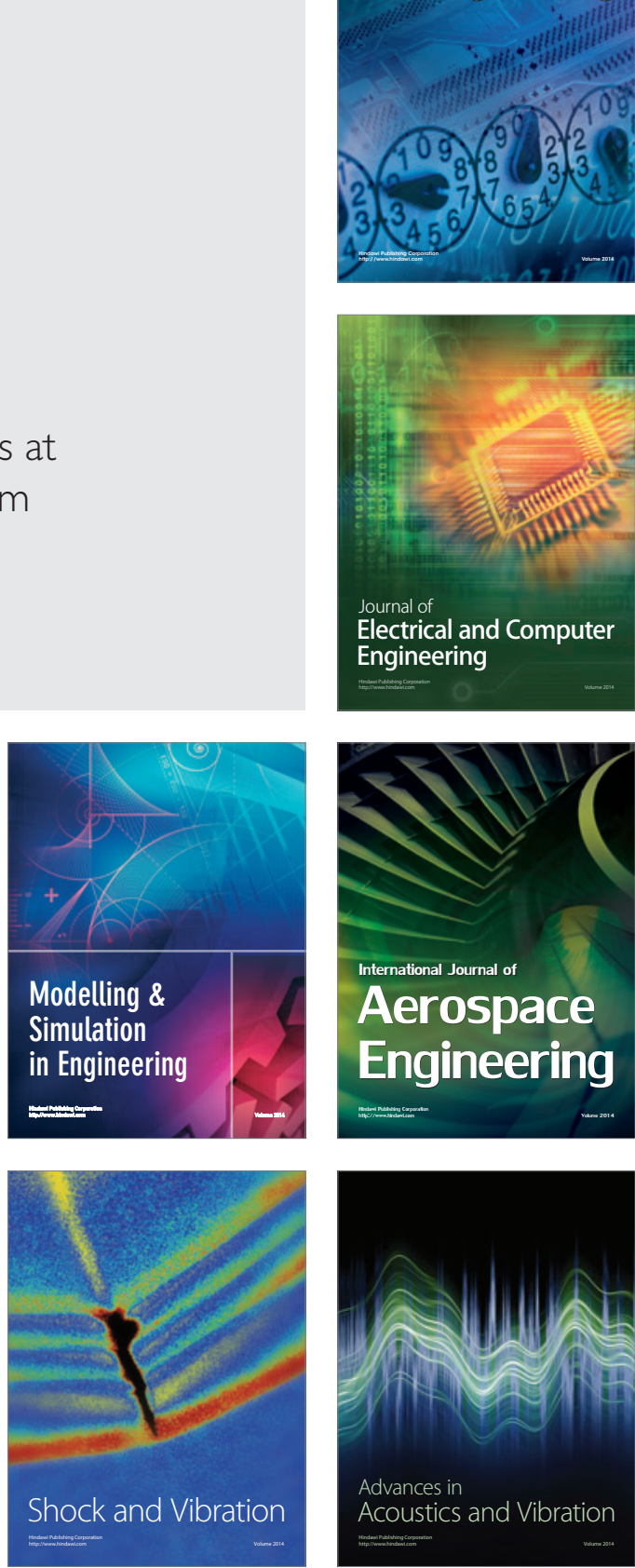